\title{
Phantom-Like Behavior of a DGP-Inspired Scalar-Gauss-Bonnet Gravity
}

\author{
Kourosh Nozari $^{a, 1}, \quad$ Tahereh Azizi ${ }^{a, 2}$ and M. R. Setare ${ }^{b, 3}$ \\ ${ }^{a}$ Department of Physics, Faculty of Basic Sciences, \\ University of Mazandaran, \\ P. O. Box 47416-95447, Babolsar, IRAN \\ ${ }^{b}$ Faculty of Science, Department of Physics, University of Kurdistan, Pasdaran Ave., Sanandaj, \\ Iran
}

\begin{abstract}
We study the phantom-like behavior of a DGP-inspired braneworld scenario where curvature correction on the brane is taken into account. We include a possible modification of the induced gravity on the brane by incorporating higher order curvature terms of Gauss-Bonnet type. We investigate the cosmological implications of the model and we show that the normal branch of the scenario self-accelerates in this modified scenario without introducing any dark energy component. Also, a phantom-like behavior can be realized in this model without introducing any phantom field that suffers from serious difficulties such as violation of the null energy condition.
\end{abstract}

PACS: 04.50.-h, 04.50.Kd, 95.36.+x

Key Words: DGP-Braneworld Scenario, Gauss-Bonnet cosmology, Phantom-like behavior

\footnotetext{
${ }^{1}$ knozari@umz.ac.ir

${ }^{2}$ t.azizi@umz.ac.ir

${ }^{3}$ rezakord@ipm.ir
} 


\section{Introduction}

Observational data indicates that our universe is currently under accelerating expansion [1]. It seems that some unknown energy components ( dark energy) with negative pressure are responsible for this late-time acceleration [2]. However, understanding the nature of dark energy is one of the fundamental problems of modern theoretical cosmology [3]. An alternative approach to accommodate dark energy is modifying the general theory of relativity on large scales. Among these theories, scalar-tensor theories [4], $f(R)$ gravity [5], DGP braneworld gravity [6] and string-inspired theories [7] are studied extensively. One interesting model of modified gravity is the Dvali-Gabadadze-Porrati (DGP) braneworld model, in which our universe is a $(3+1)$-dimensional brane embedded in an infinite Minkowski bulk. This model has a very appealing phenomenology because it predicts that $4 \mathrm{D}$ Newtonian gravity on the brane is recovered at distances shorter than a given crossover distance $r_{c}$, whereas at scale higher than $r_{c}$ the 5D effects become important due to leakage of gravity into the bulk. On the other hand, this model can explain the late-time acceleration of the universe in its self-accelerating branch without need to introducing a dark energy term [8]. Unfortunately, this branch suffers from some instabilities such as appearance of ghost degrees of freedom [9].

An alternative class of modified gravity models is the family of the string-inspired gravities by considering additional curvature invariant terms such as the Gauss-Bonnet (GB) term [10]. GB term arises naturally as the leading order of the $\alpha^{\prime}$ expansion of the heterotic string theory where, $\alpha^{\prime}$ is the inverse string tension [11]. Nojiri et al [12] showed that a particular dark energy solution can be obtained from scalar-Gauss-Bonnet cosmology. There is another version of the Gauss-Bonnet gravity namely the modified GB or $F(G)$ theory [13] that can also play the role of gravitational dark energy.

One of the essential tools for understanding the nature of dark energy is to detect the evolution of its equation of state parameter $\omega=P / \rho$, where $P$ and $\rho$ are pressure and energy density of dark energy component respectively. Recent analysis on the data from the Supernova, cosmic microwave background (CMB) and large scale structure (LSS) show that the cosmological constant fits well to the data [14], however current data also mildly favor an evolving dark energy with an equation of state $\omega$ larger than -1 in the past and less than -1 today [15] with evolution across $\omega=-1$ line in the intermediate redshift. If such a result holds on with a large number of the observational data, this would be a great challenge to the current models of dark energy. Firstly, the cosmological constant [16] as a candidate for dark energy will be excluded and dark energy must be dynamical ( note also that the cosmological constant needs a huge amount of fine-tuning). Secondly, the case with $\omega$ less than -1 which is often dubbed as phantom dark energy [17] introduces new theoretical facilities and challenges in this field. Phantom fields are a sort of scalar fields with negative sign for the kinetic energy term. In fact, phantom fields suffer from instabilities due to violation of the null energy condition, and a phantom universe eventually ends up with a Big Rip singularity [18]. Thus it seems natural to seek alternative approaches to realize a 
phantom-like behavior without introducing any phantom field in the model. Phantom-like behavior is the growth of the effective dark energy density with cosmic time and in the same time, the effective equation of state parameter should stay always less than -1 . In this regard, it has been shown that the normal, non-self-accelerating branch of the DGP scenario has the potential to explain phantom-like behavior without introducing any phantom fields on the brane ( for a number of attempts in this direction see [19]). The phantom-like behavior of 4-dimensional $f(R)$ gravity is studied in [20].

With these preliminaries, the purpose of this paper is to construct a class of DGP-inspired braneworld scenarios that curvature corrections are taken into account via incorporation of a Gauss-Bonnet type invariant term in the brane part of the action. Actually, the GaussBonnet invariant should be considered in the bulk action. However, our goal here is to consider possible modification of the induced gravity on the brane by incorporation of higher order curvature terms such as the Gauss-Bonnet type terms. In fact, since Gauss-Bonnet term is an invariant in 4-dimensions, it takes contribution in the field equations if there is a coupling between scalar degrees of freedom on the brane and this invariant term. This is the main reason for incorporation of the $\phi$ field in $f(G, \phi)$. In the previous works done by other authors in this subject, generally an effective phantom phase is realized by adopting a phantom ansatz for scale factor. Here as we will show, the phantom phase is realized on the normal branch of the DGP-inspired model with curvature correction on the brane. We note also that generally one can show, by re-construction method, that $f(R)$ gravity is equivalent to general relativity plus the scalar field [20]. We investigate the effect of higher order induced curvature correction in two alternative approaches: Scalar-Gauss-Bonnet gravity with $F(G, \phi)$ term in the brane action and modified Gauss-Bonnet gravity with $f(G)$ term that $G$ is the Gauss-Bonnet invariant.

The paper is organized as follows: In section 2 we start from the action of the DGPinspired $F(G, \phi)$ scenario and derive the corresponding field equations. Then, we study the cosmological implications of this model. We show that in the presence of the curvature correction due to a Scalar-Gauss-Bonnet term in the action, the normal branch of the scenario has a self-accelerating behavior. The expansion history of this model will be studied and a comparison between luminosity distances in this model, the $\Lambda$ DGP and $\Lambda$ CDM will be performed. On the other hand, in this scenario we obtain a phantom-like behavior without introducing a phantom field neither in the bulk nor on the brane. In section 3 we consider a modified Gauss-Bonnet gravity in the DGP braneworld and we obtain the cosmological equations of the scenario. We show that this scenario can also realize a phantom-like behavior by adopting viable ansatz for the scale factor in the appropriate subspaces of the model parameter space. Finally, our summery and conclusions are presented in section 4 . 


\section{A DGP-Inspired $F(G, \phi)$ Scenario}

We start with the action of a DGP-inspired $F(G, \phi)$ scenario as follows

$$
\mathcal{S}=\int_{\text {bulk }} d^{5} X \sqrt{-{ }^{(5)} g} \frac{m_{5}^{3}}{2} \mathcal{R}+\int_{\text {brane }} d^{4} x \sqrt{-g}\left[\frac{m_{4}^{2}}{2} R+m_{5}^{3} \bar{K}+F(G, \phi)+\mathcal{L}_{m}\right] .
$$

Here $X^{A}$ with $A=0,1,2,3,5$ are coordinates in the bulk, while $x^{\mu}$ with $\mu=0,1,2,3$ are induced coordinates on the brane. $m_{5}^{3}$ is the 5 -dimensional Planck mass and $\mathcal{R}$ is the 5 dimensional Ricci scalar. Also, $m_{4}^{2}$ is the 4-dimensional Planck mass and $\bar{K}$ is the trace of the mean extrinsic curvature on the brane in the higher dimensional bulk, corresponding to the York-Gibbons-Hawking boundary term [21] defined as

$$
\bar{K}_{\mu \nu}=\frac{1}{2} \lim _{\epsilon \rightarrow+0}\left(\left[K_{\mu \nu}\right]_{y=-\epsilon}+\left[K_{\mu \nu}\right]_{y=+\epsilon}\right) .
$$

$y$ is the coordinate of the extra dimension and the brane is located at $y=0$. $R$ is the induced Ricci scalar on the brane, and the Scalar-Gauss-Bonnet term is defined as

$$
F(G, \phi) \equiv-\frac{1}{2} \partial_{\mu} \phi \partial^{\mu} \phi-V(\phi)+f(\phi) G(R)
$$

By definition, the Gauss-Bonnet invariant $G(R)$ is given by

$$
G(R)=R^{2}-4 R_{\mu \nu} R^{\mu \nu}+R_{\mu \nu \alpha \beta} R^{\mu \nu \alpha \beta}
$$

where all terms on the right hand side are functions of the induced curvature on the brane. In the action $(1), \mathcal{L}_{m}$ is the Lagrangian of the other matters localized on the brane. It is important to note that in the matter action, the matter is minimally coupled to the metric and not to the scalar field, making the Gauss-Bonnet gravity a metric theory which leads to conservation of matter on the brane. Note also that we have incorporated possible modification of the induced gravity on the brane by inclusion of the higher order induced curvature terms via the Gauss-Bonnet invariant. The corresponding energy-momentum tensor $T_{\mu \nu}^{(m)}$ is defined as

$$
T_{\mu \nu}^{(m)}=-\frac{2}{\sqrt{-g}} \frac{\delta\left(\sqrt{-g} \mathcal{L}_{m}\right)}{\delta g^{\mu \nu}} .
$$

Variation of the action (1) with respect to the scalar field, gives the equation of motion for the scalar field on the brane,

$$
\nabla^{2} \phi-V^{\prime}(\phi)+f^{\prime}(\phi) G(R)=0
$$

where the prime denotes a derivative with respect to the scalar field $\phi$. On the other hand, the bulk-brane Einstein's equations calculated from the action (1) are given by

$$
m_{5}^{3}\left(\mathcal{R}_{A B}-\frac{1}{2} g_{A B} \mathcal{R}\right)+m_{4}^{2} \delta_{A}^{\mu} \delta_{B}^{\nu}\left[R_{\mu \nu}-\frac{1}{2} g_{\mu \nu} R-\frac{1}{2} g^{\mu \nu} f(\phi) G(R)+2 f(\phi) R R^{\mu \nu}\right.
$$




$$
\left.+4 f(\phi) R_{\rho}^{\mu} R^{\nu \rho}+2 f(\phi) R^{\mu \rho \sigma \tau} R_{\rho \sigma \tau}^{\mu}-4 f(\phi) R^{\mu \rho \sigma \nu} R_{\rho \sigma}\right] \delta(y)=\delta_{A}{ }^{\mu} \delta_{B}{ }^{\nu} \tau_{\mu \nu} \delta(y),
$$

where

$$
\tau^{\mu \nu} \equiv T_{(M)}^{\mu \nu}+T_{(\phi)}^{\mu \nu}+T_{(c)}^{\mu \nu} .
$$

The energy-momentum tensor corresponding to the scalar field and curvature are defined as $[12]$

$$
T_{(\phi)}^{\mu \nu}=\frac{1}{2} \partial^{\mu} \phi \partial^{\nu} \phi-\frac{1}{4} g^{\mu \nu} \partial_{\rho} \phi \partial^{\rho} \phi-\frac{1}{2} g^{\mu \nu} V(\phi),
$$

and

$$
\begin{gathered}
T_{(c)}^{\mu \nu}=2\left[\nabla^{\mu} \nabla^{\nu} f(\phi)\right] R-2 g^{\mu \nu}\left[\nabla^{2} f(\phi)\right] R-4\left[\nabla_{\rho} \nabla^{\mu} f(\phi)\right] R^{\nu \rho} \\
\left.-4\left[\nabla_{\rho} \nabla^{\nu} f(\phi)\right] R^{\mu \rho}+4\left[\nabla^{2} f(\phi)\right] R^{\mu \nu}+4 g^{\mu \nu}\left[\nabla_{\rho} \nabla_{\sigma} f(\phi)\right] R^{\rho \sigma}-4 \nabla_{\rho} \nabla_{\sigma} f(\phi)\right] R^{\mu \rho \sigma \nu}
\end{gathered}
$$

respectively. Equation (6) can be rewritten as follows

$$
m_{4}^{3}\left(\mathcal{R}_{A B}-\frac{1}{2} g_{A B} \mathcal{R}\right)+m_{3}^{2} \delta_{A}{ }^{\mu} \delta_{B}{ }^{\nu}\left(R_{\mu \nu}-\frac{1}{2} g_{\mu \nu} R\right) \delta(y)=\delta_{A}{ }^{\mu} \delta_{B}{ }^{\nu} \mathcal{T}_{\mu \nu} \delta(y)
$$

where $\mathcal{T}_{\mu \nu}$ is the total energy-momentum on the brane defined as $\mathcal{T}_{\mu \nu}=-\frac{1}{2} g^{\mu \nu} f(\phi) G(R)+2 f(\phi) R R^{\mu \nu}+4 f(\phi) R_{\rho}^{\mu} R^{\nu \rho}+2 f(\phi) R^{\mu \rho \sigma \tau} R_{\rho \sigma \tau}^{\mu}-4 f(\phi) R^{\mu \rho \sigma \nu} R_{\rho \sigma}+\tau_{\mu \nu}$.

From equation (10) we find

$$
G_{A B}=\mathcal{R}_{A B}-\frac{1}{2} g_{A B} \mathcal{R}=0
$$

and

$$
G_{\mu \nu}=R_{\mu \nu}-\frac{1}{2} g_{\mu \nu} R=\frac{1}{m_{4}^{2}} \mathcal{T}_{\mu \nu} .
$$

for bulk and brane respectively. The corresponding junction conditions relating the extrinsic curvature to the energy-momentum tensor of the brane, have the following form

$$
\lim _{\epsilon \rightarrow+0}\left[K_{\mu \nu}\right]_{y=-\epsilon}^{y=+\epsilon}=\frac{1}{m_{5}^{3}}\left[\mathcal{T}_{\mu \nu}-\frac{1}{3} g_{\mu \nu} g^{\alpha \beta} \mathcal{T}_{\alpha \beta}\right]_{y=0}-\frac{m_{4}^{2}}{m_{5}^{3}}\left[R_{\mu \nu}-\frac{1}{6} g_{\mu \nu} g^{\alpha \beta} R_{\alpha \beta}\right]_{y=0}
$$

Using the 5D Codacci equation, the field equation of the bulk and the junction condition at the brane lead to the conservation of the total energy momentum tensor of the brane so that

$$
\nabla^{\nu} \mathcal{T}_{\mu \nu}=0
$$

Note that, the matter sector of the energy momentum tensor $T_{\mu \nu}^{(m)}$ can be described by a perfect fluid with energy density $\rho^{(m)}$ and pressure $P^{(m)}$ and satisfies the continuity equation by virtue of the Bianchi identity

$$
\dot{\rho}^{(m)}+3 H\left(1+\omega^{(m)}\right) \rho^{(m)}=0 .
$$




\subsection{Cosmological Implications of the model}

To study the cosmology of a homogenous and isotropic brane in this setup, we consider the following line element

$$
d s^{2}=g_{\mu \nu} d x^{\mu} d x^{\nu}+b^{2}(y, t) d y^{2}=-n^{2}(y, t) d t^{2}+a^{2}(y, t) \gamma_{i j} d x^{i} d x^{j}+b^{2}(y, t) d y^{2} .
$$

In this relation, $\gamma_{i j}$ is a maximally symmetric 3 -dimensional metric defined as $\gamma_{i j}=\delta_{i j}+$ $k \frac{x_{i} x_{j}}{1-k r^{2}}$ where $k=-1,0,1$ corresponding to possible spatial geometries of the brane and $r^{2}=x_{i} x^{i}$. Now, choosing a Gaussian normal coordinate system so that $b^{2}(y, t)=1$, leads to the following form of the junction condition (14) on the brane with the non-vanishing components of the Einstein's tensor in the bulk

$$
\lim _{\epsilon \rightarrow+0}\left[\partial_{y} n\right]_{y=-\epsilon}^{y=+\epsilon}(t)=\frac{2 n m_{4}^{2}}{m_{5}^{3}}\left[\frac{\ddot{a}}{n^{2} a}-\frac{\dot{a}^{2}}{2 n^{2} a^{2}}-\frac{\dot{n} \dot{a}}{n^{3} a}-\frac{k}{2 a^{2}}\right]_{y=0}+\frac{n}{3 m_{5}^{3}}\left[2 \rho^{(t o t)}+3 P^{(t o t)}\right]_{y=0},
$$

and

$$
\lim _{\epsilon \rightarrow+0}\left[\partial_{y} a\right]_{y=-\epsilon}^{y=+\epsilon}(t)=\frac{m_{4}^{2}}{m_{5}^{3}}\left[\frac{\dot{a}^{2}}{n^{2} a}+\frac{k}{a}\right]_{y=0}-\left[\frac{\rho^{(t o t)} a}{2 m_{5}^{3}}\right]_{y=0} .
$$

With these equations, the generalized Friedmann equation for the cosmological dynamics on the brane can be written as follows

$$
m_{4}^{2}\left[H^{2}+\frac{k}{a^{2}}-\frac{8 \pi G\left(\rho^{(m)}+\rho^{(G B)}\right)}{3}\right]^{2}=m_{5}^{6}\left(H^{2}+\frac{k}{a^{2}}-\frac{C}{a^{4}}\right) .
$$

where the last term in the right hand side of this equation represents the dark radiation term and $C$ is an integration constant. It is important to note that this term can be neglected at present time due to its fast decaying behavior. The energy density corresponding to the Gauss-Bonnet term is defined as

$$
\rho^{(G B)} \equiv \frac{1}{2} \dot{\phi}^{2}+V(\phi)-24 \dot{\phi} f^{\prime}(\phi) H^{3}
$$

and the corresponding pressure is defined as

$$
P^{(G B)}=\frac{1}{2} \dot{\phi}^{2}-V(\phi)+8 \frac{\partial}{\partial t}\left(H^{2} \dot{f}\right)+16 H^{3} \dot{\phi} f^{\prime}(\phi),
$$

where a dot refers to derivative with respect to the cosmic time of the brane. The modified Friedmann equation (20) can be rewritten as follows

$$
H^{2}+\frac{k}{a^{2}}=\frac{\rho^{(m)}+\rho^{(G B)}}{3 m_{4}^{2}}+\frac{1}{2 r_{c}^{2}}+\varepsilon \sqrt{\frac{1}{4 r_{c}^{4}}+\frac{1}{r_{c}^{2}}\left(\frac{\rho^{(m)}+\rho^{(G B)}}{3 m_{4}^{2}}\right)} .
$$

where $\varepsilon= \pm 1$ is associated to two possible branches of the scenario corresponding to two different embeddings of the brane in the bulk and $r_{c}=\frac{m_{4}^{2}}{2 m_{5}^{3}}$ is the crossover scale. In the 
distance scale lower than this scale, gravity behaves as usual 4D scenario but in the distance scales higher than the crossover scale, gravity leaks to the extra dimension and this leakage leads to weakness of gravity in the large scales, so the universe accelerates. In other words, weakness of gravity at large scales plays the role of a negative pressure fluids that accelerates the universe expansion. Equation (23) as the modified Friedmann equation in this DGPinspired $F(G, \phi)$ scenario is similar to the standard induced gravity result, but we note that here there is an important difference which is hidden in the definition of the total energy density and pressure on the brane. There is a contribution from higher order curvature corrections in these total quantities and hence in the modified Friedmann equation (23). In another words, the effect of the higher order curvature corrections in the brane action are interpreted as a contribution in the total energy density and pressure on the brane. On the other hand, the modified Raychaudhuri equation in this setup has the following form

$$
\begin{gathered}
\left\{1+\frac{1}{3 m_{4}^{2}} r_{c}\left[\rho^{(m)}+\rho^{(G B)}-6 r_{c}\left(H^{2}+\frac{k}{a^{2}}\right)\right]\right\}\left(\dot{H}-\frac{k}{a^{2}}\right)=-\frac{1}{12 m_{4}^{2}}\left(\rho^{(m)}+\rho^{(G B)}+P^{(m)}+P^{(G B)}\right) \\
\times\left[\rho^{(m)}+\rho^{(G B)}-6 r_{c}\left(H^{2}+\frac{k}{a^{2}}\right)\right] .
\end{gathered}
$$

\subsection{Self-accelerating the normal branch with curvature correction}

In which follows we assume a spatially flat FRW brane. In this case, we can rewrite the modified Friedmann equation (23) in the following form

$$
H^{2}=\frac{8 \pi G}{3}\left(\rho^{(m)}+\rho^{(G B)}\right)+\varepsilon \frac{H}{r_{c}} .
$$

where $G \equiv 1 / 8 \pi m_{4}^{2}$ is the $4 \mathrm{D}$ gravitational constant. One of the simplest cosmological model that can exhibit acceleration of the universe is a de Sitter spacetime. So, it seems interesting to looking for such solutions in our model. With this motivation, we can probe the selfaccelerating solution of the modified normal branch of this scenario. Assuming a de Sitter spacetime, i.e., looking for solutions of the form $a(t)=a_{0} \exp \left(H_{0} t\right)$, where $H_{0}$ and $a_{0}$ are constants, the modified Friedmann equation (25) in a de Sitter universe with Hubble rate $H_{0}$ is given by

$$
\rho^{(m)}+V_{0}=\text { constant }
$$

where the subscript 0 stands for quantities evaluated in the de Sitter phase and the scalar field is assumed to be constant in time. This relation shows that the matter energy density is constant by the virtue of the continuity equation (16), leads to a cosmological constant behavior for the matter sector $\left(\omega^{(m)}=-1\right)$ on the brane. In which follows, we neglect the matter content of the brane in our analysis of de Sitter brane. Using equation (23), the Hubble rate can be expressed as follows

$$
2 r_{c}^{2} H_{0}^{2}=1+\frac{16 \pi G r_{c}^{2} V_{0}}{3}+\varepsilon \sqrt{1+\frac{32 \pi G r_{c}^{2}}{3} V_{0}} .
$$


Note that the DGP braches can be recovered by imposing $V_{0}=0$. Indeed, the case $\varepsilon=1$ branch is corresponding to the de Sitter self-accelerating solution, and the $\varepsilon=-1$ case corresponds to the normal branch leads to a Minkowski spacetime or the non-self-accelerating solution. In the presence of the curvature correction due to a Scalar-Gauss-Bonnet term in the action, the normal branch of the scenario self-accelerates too. It is important to note that the normal branch has not the difficulties of the self-accelerating one such as the ghost instabilities. In figure 1, we plot the behavior of the re-scaled squared Hubble parameter $2 r_{c}^{2} H_{0}^{2}$ versus the re-scaled energy density $\frac{16 \pi G r_{c}^{2}}{3} V_{0}$ for two branches of the solutions. This figure shows that the normal branch of the scenario exhibits a self-accelerating behavior because of the presence of an extra energy density originated from curvature corrections.
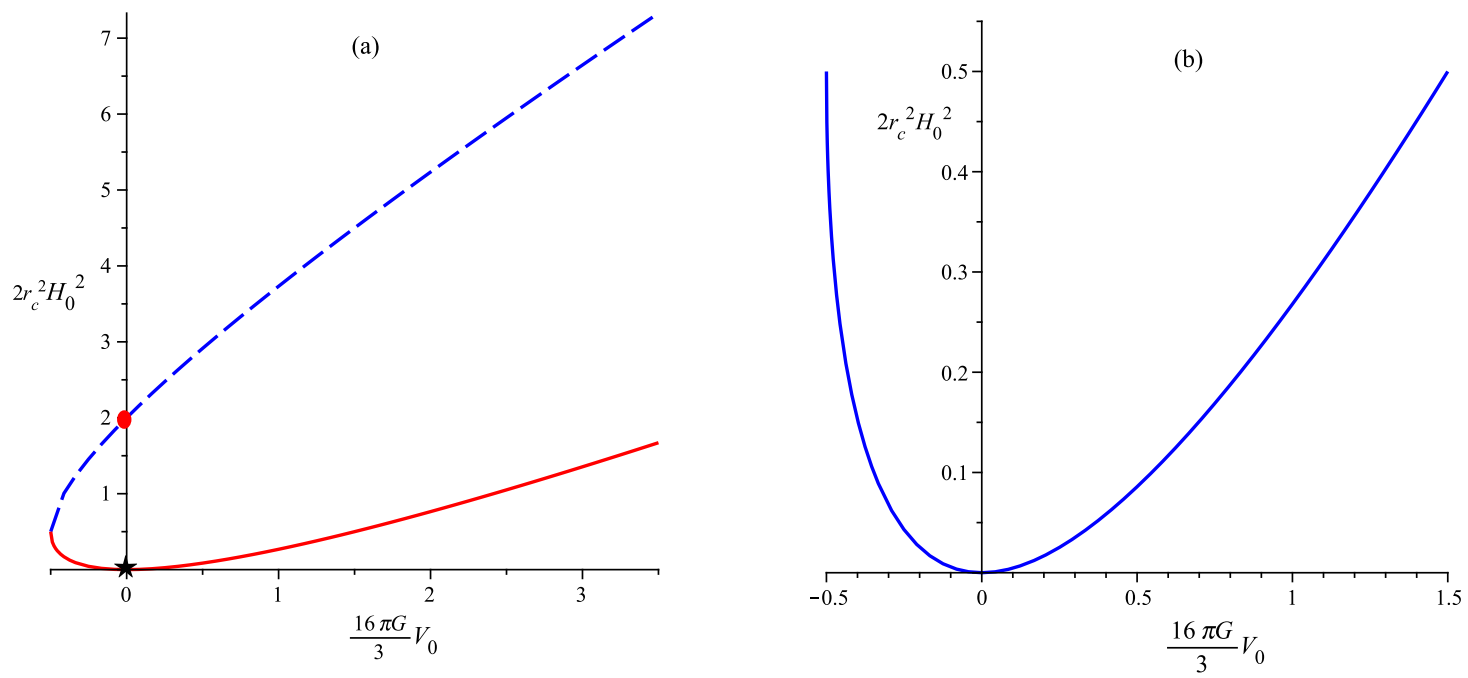

Figure 1: (a) Behavior of the re-scaled squared Hubble parameter $2 r_{c}^{2} H_{0}^{2}$ versus the re-scaled energy density $\frac{16 \pi G r_{c}^{2}}{3} V_{0}$ for two branches of the scenario. The solid curve (with star) is corresponding to the normal branch and the dashed curve ( with circle) corresponds to the self-accelerating branch of the scenario. (b) A zoom on the normal branch of the DGP scenario with curvature effect.

Up to this point, we have focused on the effects of a curvature correction term on the DGP model. Now we consider the effect of higher order embedding on the 4D Scalar-GaussBonnet model. In other words, we study the effect of extra dimension on the cosmological dynamics on the brane. The Hubble parameter (27) can be expressed in a $4 \mathrm{D}$ regime as follows

$$
H_{0}^{2}=H_{(4)}^{2}+\frac{1+\varepsilon \sqrt{1+\frac{32 \pi G r_{c}^{2}}{3} V_{0}}}{2 r_{c}^{2}} .
$$

where $H_{(4)}^{2}=\frac{8 \pi G}{3} V_{0}$. This relation shows that the effect of an extra dimension is a shift on the $4 \mathrm{D}$ Hubble rate. In order to de Sitter brane to be close to the standard 4D regime ( with 
$\left.H_{(0)}^{2} \sim H_{(4)}^{2}\right)$, the following condition should be satisfied

$$
\left|\frac{3\left(1+\varepsilon \sqrt{1+\frac{32 \pi G r_{c}^{2}}{3} V_{0}}\right)}{16 \pi G r_{c}^{2} V_{0}}\right| \ll 1
$$

\subsection{Phantom-Like Mimicry}

We can express the Friedmann equation (23) for the normal branch of this DGP-inspired scenario in a dimensionless form as follows

$$
\begin{aligned}
E^{2} & =\frac{H^{2}(z)}{H_{0}^{2}}=\Omega_{m}(1+z)^{3}+\Omega_{G B}(1+z)^{3\left(1+\omega_{G B}\right)}+2 \Omega_{r_{c}} \\
& -2 \sqrt{\Omega_{r_{c}}} \sqrt{\Omega_{m}(1+z)^{3}+\Omega_{G B}(1+z)^{3\left(1+\omega_{G B}\right)}+\Omega_{r_{c}}}
\end{aligned}
$$

where $\Omega_{m}=\frac{8 \pi G \rho_{0}^{(m)}}{3 H_{0}^{2}}, \Omega_{G B}=\frac{8 \pi G \rho_{0}^{(G B)}}{3 H_{0}^{2}}$ and $\Omega_{r_{c}}=\frac{1}{4 r_{c}^{2} H_{0}^{2}}$. Now we study the constraints imposed on this model. At redshift $z=0$, equation (30) can be expressed as ( see Ref. [22] for a general framework in this direction)

$$
1=\left(\sqrt{\Omega_{m}+\Omega_{G B}+\Omega_{r_{c}}}-\sqrt{\Omega_{r_{c}}}\right)^{2} .
$$

This expression indicates that $\Omega_{r_{c}}$ should be positive and $\Omega_{m}+\Omega_{G B}+\Omega_{r_{c}}>0$ too. Taking the square root of the last equation, there are two possibility for the sign of the quantity $\Omega_{m}+\Omega_{G B}$ as follows

$$
\Omega_{m}+\Omega_{G B} \geq 0
$$

and

$$
-\Omega_{r_{c}} \leq \Omega_{m}+\Omega_{G B}<0
$$

In the first case, taking the square root of the equation (31) yields

$$
1+\sqrt{\Omega_{r_{c}}}=\sqrt{\Omega_{m}+\Omega_{G B}+\Omega_{r_{c}}} .
$$

Now by squaring both sides of this equation we find

$$
\Omega_{m}+\Omega_{G B}-2 \sqrt{\Omega_{r_{c}}}=1 .
$$

Similarly, for the second case the constraint equation is

$$
\Omega_{m}+\Omega_{G B}+2 \sqrt{\Omega_{r_{c}}}=1 .
$$

Note that the general relativistic limit can be recovered if we set $\Omega_{r_{c}}=0$ (or $m_{5}=0$ ). In this case equation (31) implies that $\Omega_{m}+\Omega_{G B}=1$. 
For a comparison between this DGP-inspired $F(G, \phi)$ scenario and the well-known $\Lambda$ DGP ( see for instance [23]) and $\Lambda$ CDM models, we study the expansion histories of these alternative scenarios. We focus on the variation of the Luminosity Distance versus the redshift in these scenarios. The luminosity distance for a spatially flat universe is expressed as follows

$$
d_{L}(z)=(1+z) \int_{0}^{z} \frac{d z}{H(z)},
$$

where for the DGP-inspired $F(G, \phi)$ scenario, the Hubble rate is given by equation (30). For $\Lambda$ DGP model, $H(z)$ is defined as [23]

$$
\frac{H(z)}{H_{0}}=\frac{1}{2}\left[-\frac{1}{r_{0} H_{0}}+\sqrt{\left(2+\frac{1}{r_{0} H_{0}}\right)^{2}+4 \Omega_{M}^{0}\left[(1+z)^{3}-1\right]}\right] .
$$

The luminosity distance of the $\Lambda \mathrm{CDM}$ model is given by

$$
d_{L}^{\Lambda C D M}(z)=(1+z) \int_{0}^{z} \frac{H_{0} d z}{\sqrt{\Omega_{m}(1+z)^{3}+\Omega_{\Lambda}}} .
$$

In figure (2) we show that the luminosity distance of the DGP-inspired scenario with curvature correction is closer to the $\Lambda \mathrm{CDM}$ model than the $\Lambda \mathrm{DGP}$ scenario. We note that this result is dependent on the choice of the model parameter space. Nevertheless, since the $\Lambda \mathrm{CDM}$ has a very good agreement with observational data, our analysis shows that with a suitable choice of the parameter space of the model, the DGP-inspired $F(G, \phi)$ scenario has better agreement with recent observation than $\Lambda$ DGP. This result seems to be reasonable since the DGP-inspired $F(G, \phi)$ scenario has wider parameter space than the $\Lambda$ DGP model.

Now, we show that our model can lead to the phantom-like behavior on the brane without need to introduce any kind of the phantom scalar fields that violate the null energy condition. For this goal, the standard Friedman equation can be written as an effective form

$$
H^{2}=\frac{8 \pi G}{3}\left(\rho^{(m)}+\rho_{\text {eff }}^{(D E)}\right)
$$

where $\rho^{(m)}$ is the energy density of the standard matter and $\rho_{e f f}^{(D E)}$ is the energy density corresponding to dark energy. Comparison between the normal branch of equation (20) with this equation leads to the following relation for $\rho_{\text {eff }}^{(D E)}$

$$
\frac{8 \pi G}{3} \rho_{e f f}^{(D E)}=\rho^{(G B)}+\frac{3}{2 r_{c}^{2}}\left[1-\sqrt{1+\frac{32 \pi G r_{c}^{2}}{3}\left(\rho^{(m)}+\rho^{(G B)}\right)}\right]
$$

where we have set $8 \pi G=1$. With the observable quantities, this equation is given by

$$
\frac{\rho_{e f f}^{(D E)}}{H_{0}^{2}}=\Omega_{G B}(1+z)^{3\left(1+\omega_{G B}\right)}+6 \Omega_{r_{c}}-6 \sqrt{\Omega_{r_{c}}} \sqrt{\Omega_{m}(1+z)^{3}+\Omega_{G B}(1+z)^{3\left(1+\omega_{G B}\right)}+\Omega_{r_{c}}}
$$




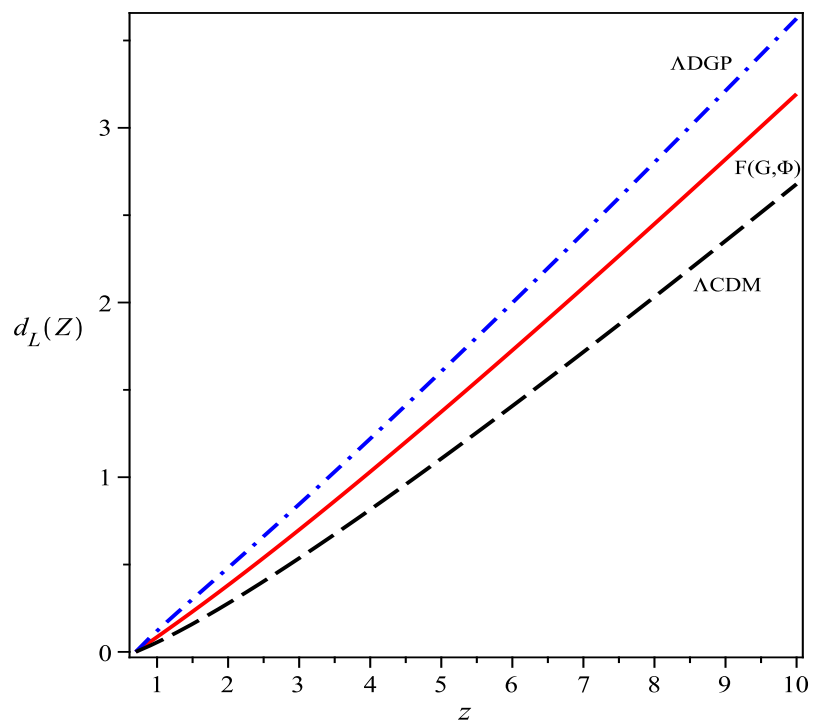

Figure 2: Luminosity distance versus the redshift for three alternative scenarios. In this figure we have set $\Omega_{m}=0.25, \Omega_{r c}=0.12$, and $\Omega_{G B}=-0.56$. With these values, we calculate $\Omega_{G B}$ using the constraint equation (35). There is better agreement between DGP-inspired $F(G, \phi)$ scenario and the $\Lambda \mathrm{CDM}$ at least in the parameter values adopted here.

Note that $\rho_{e f f}^{(D E)}$ and $\omega_{e f f}=\frac{P_{e f f}}{\rho_{e f f}}$ satisfy the continuity equation in the same way as the general relativity

$$
\dot{\rho}_{e f f}^{(D E)}+3 H\left(1+\omega_{e f f}\right) \rho_{e f f}^{(D E)}=0 .
$$

In this respect, the effective equation of state of dark energy can be expressed as follows

$$
\omega_{e f f}=-1+\frac{\left(1-\sqrt{\Omega_{r_{c}}}\right) \Omega_{G B}(1+z)^{3\left(1+\omega_{G B}\right)}-\sqrt{\Omega_{r_{c}}} \Omega_{m}(1+z)^{3}}{\rho_{e f f}^{(D E)} \sqrt{\Omega_{m}(1+z)^{3}+\Omega_{G B}(1+z)^{3\left(1+\omega_{G B}\right)}+\Omega_{r_{c}}}}
$$

In figure 3 we plot the effective energy density and equation of state parameter of the model versus the redshift $z$. This figure shows a growing behavior of the effective energy density with decreasing of $z$; a requirement for phantom-like prescription. On the other hand, the effective equation of state parameter remains less than -1 in the phantom phase at future. Especially, the figure shows that the effective equation of state parameter has passed the phantom divide line $\omega_{e f f}=-1$ in the near past. From figure 4 we can obtain appropriate ranges of $z$ to fulfill the null energy condition, $\left(\rho_{\text {eff }}^{(D E)}+P_{\text {eff }}^{(D E)} \geq 0\right)$. Clearly, this condition is satisfied in the present and future times at least in some subspaces of the model parameter space. As a result, the phantom-like prescription is realized in this DGP-inspired scenario without introduction of any phantom field neither in the bulk nor on the brane. 

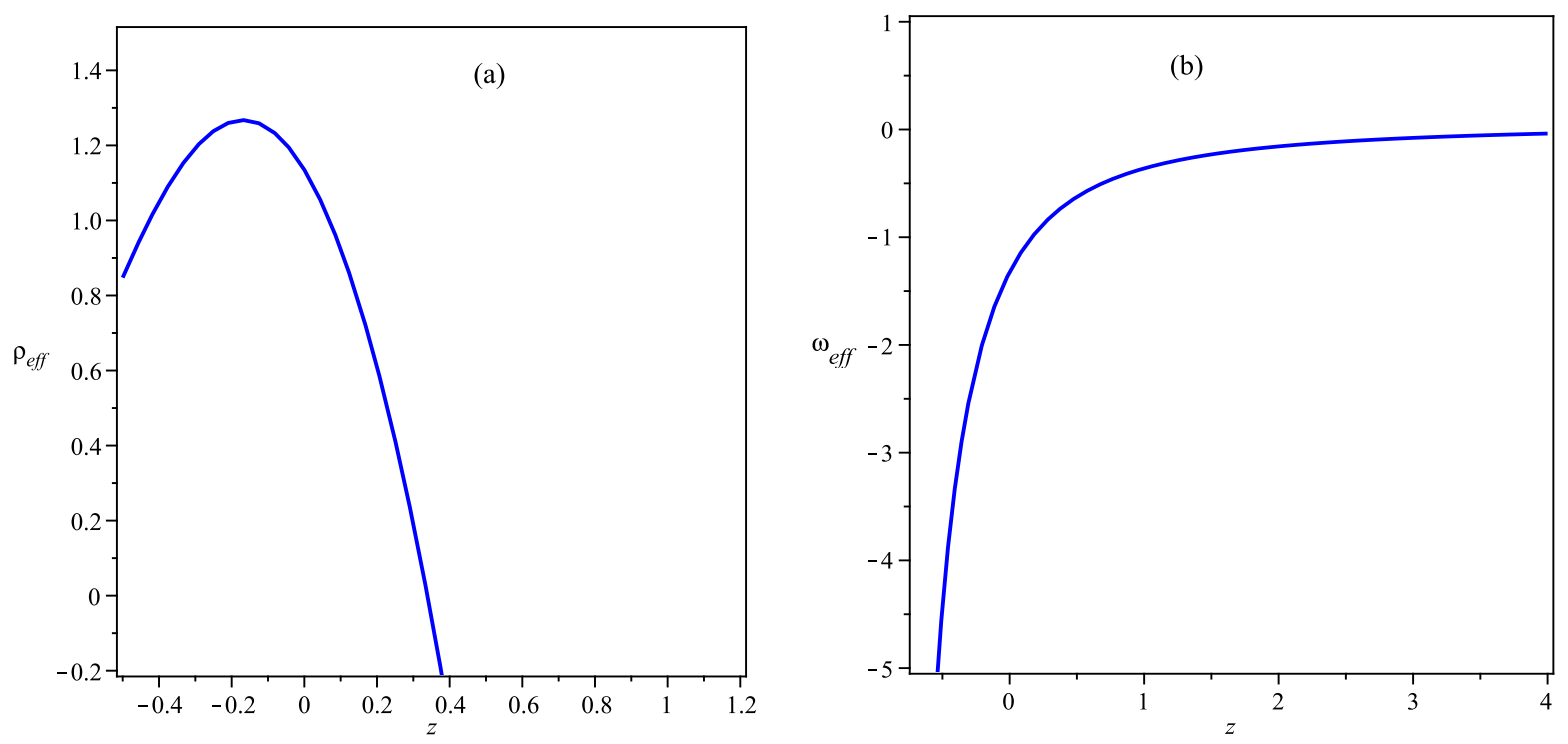

Figure 3: a) Variation of the effective dark energy density versus the redshift. The effective dark energy density grows by decreasing $z$ and therefore shows a phantom-like behavior. b) The effective equation of state parameter versus the redshift. The braneworld universe has entered in the phantom phase in the near past and there is a smooth crossing of the phantom divide line in this setup.

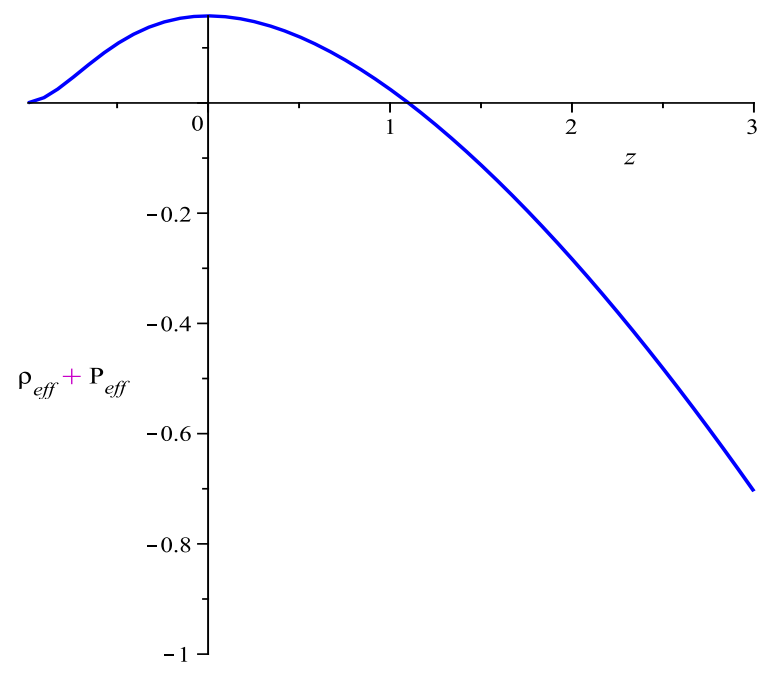

Figure 4: The null energy condition is fulfilled for $z<1.1$. 


\section{An Extension with $f(G)$ Term on the Brane}

In this section we consider a modified Gauss-Bonnet (modified-GB) term on the action of the DGP brane. The modified-GB gravity has been extensively studied in the literature ( see for example [13] ). Especially in Ref. [24] it has been shown that this model may play the role of a gravitational alternative to dark energy proposal. On the other hand, the modified-GB scenario has no ghosts and is stable, and it also passes the solar system tests. The action of the DGP-inspired modified Gauss-Bonnet gravity is given by

$$
\mathcal{S}=\int_{\text {bulk }} d^{5} X \sqrt{-{ }^{(5)} g} \frac{m_{5}^{3}}{2} \mathcal{R}+\int_{\text {brane }} d^{4} x \sqrt{-g}\left[\frac{m_{4}^{2}}{2} R+m_{5}^{3} \bar{K}+f(G)+\mathcal{L}_{m}\right] .
$$

Note that the brane part of the action is dynamically equivalent to the Scalar-Gauss-Bonnet gravity with vanishing kinetic-energy term (see [24] for more details). In this action, $f(G)$ is an arbitrary function of the Gauss-Bonnet invariant which is defined in equation (3). In the same line as the previous section, the bulk-brane Einstein's equations calculated from action (45) are given by

$$
m_{4}^{3}\left(\mathcal{R}_{A B}-\frac{1}{2} g_{A B} \mathcal{R}\right)+m_{3}^{2} \delta_{A}{ }^{\mu} \delta_{B}{ }^{\nu}\left(R_{\mu \nu}-\frac{1}{2} g_{\mu \nu} R\right) \delta(y)=\delta_{A}{ }^{\mu} \delta_{B}{ }^{\nu} \mathcal{T}_{\mu \nu} \delta(y),
$$

where $\mathcal{T}_{\mu \nu}$ is the total energy-momentum on the brane defined as $\mathcal{T}_{\mu \nu}=T_{\mu \nu}^{(m)}+T_{\mu \nu}^{(f)}$. The energy-momentum tensor $T_{\mu \nu}^{(f)}$ is corresponding to the Gauss-Bonnet curvature on the brane and is given by

$$
\begin{gathered}
T^{\mu \nu(f)}=\frac{1}{2} g^{\mu \nu} f(G)-2 f^{\prime}(G) R R^{\mu \nu}+4 f^{\prime}(G) R_{\rho}^{\mu} R^{\nu \rho}-2 f^{\prime}(G) R^{\mu \rho \sigma \tau} R_{\rho \sigma \tau}^{\nu}-4 f^{\prime}(G) R^{\mu \rho \sigma \nu} R_{\rho \sigma} \\
+2\left[\nabla^{\mu} \nabla^{\nu} f^{\prime}(G)\right] R-2 g^{\mu \nu}\left[\nabla^{2} f^{\prime}(G)\right] R-4\left[\nabla_{\rho} \nabla^{\mu} f^{\prime}(G)\right] R^{\nu \rho}-4\left[\nabla_{\rho} \nabla^{\nu} f^{\prime}(G)\right] R^{\mu \rho}+4\left[\nabla^{2} f^{\prime}(G)\right] R^{\mu \nu} \\
\left.+4 g^{\mu \nu}\left[\nabla_{\rho} \nabla_{\sigma} f^{\prime}(G)\right] R^{\rho \sigma}-4 \nabla_{\rho} \nabla_{\sigma} f^{\prime}(G)\right] R^{\mu \rho \sigma \nu}
\end{gathered}
$$

where the prime denotes a derivative with respect to the $G$. From the equation (46), the field equations can be deduced as follows

$$
G_{A B}=\mathcal{R}_{A B}-\frac{1}{2} g_{A B} \mathcal{R}=0
$$

and

$$
G_{\mu \nu}=R_{\mu \nu}-\frac{1}{2} q_{\mu \nu} R=\frac{1}{m_{4}^{2}} \mathcal{T}_{\mu \nu} .
$$

for the bulk and brane respectively. By imposing the junction conditions and using the FRW metric on the brane ( equations (17)-(19)), we find the following Friedmann equation for the normal branch of the scenario

$$
H^{2}=\frac{1}{3}\left(\rho^{(m)}+\rho^{(f)}\right)-\frac{H}{r_{c}} .
$$


Where we have set $m_{4}^{2} \equiv 1$. The energy density and pressure corresponding to the GaussBonnet term can be defined as [22]

$$
\rho^{(f)}=G f^{\prime}(G)-f(G)-24^{2} H^{4}\left(2 \dot{H}^{2}+H \ddot{H}+4 H^{2} \dot{H}\right) f^{\prime \prime}(G)
$$

and

$$
P^{(f)}=f(G)-24^{2} H^{2}\left(3 H^{4}+20 H^{2} \dot{H}^{2}+6 \dot{H}^{3}+4 H^{3} \ddot{H}+H^{2} \ddot{H}\right) f^{\prime \prime}(G)
$$

respectively. Comparing equation (50) with the standard Friedmann equation, we can define an effective energy density as follows

$$
H^{2}=\frac{1}{3}\left(\rho^{(m)}+\rho_{e f f}^{(D E)}\right)
$$

where $\rho^{(m)}$ is the energy density of the standard matter and $\rho_{\text {eff }}^{(D E)}$ corresponds to the energy density of dark energy which is given by

$$
\rho_{\text {eff }}^{(D E)}=\rho^{(f)}-\frac{3 H}{r_{c}} .
$$

Now we assume $f(G)$ to be defined as $f(G) \equiv f_{0}|G|^{\beta}$ with constants $f_{0}$ and $\beta$. For $\beta<\frac{1}{2}$, the $f(G)$ term becomes dominant in the small curvature regime. We note that $\beta$ can take essentially both positive and negative signs. Now the effective energy density can be rewritten in the following form

$$
\rho_{\text {eff }}^{(D E)}=f_{0} G^{\beta}\left(f_{0} \beta-1-\left(\frac{24 \beta H^{2}}{G}\right)^{2}\left[2 \dot{H}^{2}+H \ddot{H}+4 H^{2} \dot{H}\right]\right)-\frac{3 H}{r_{c}} .
$$

As usual, the effective quantities $\rho_{e f f}^{(D E)}$ and $\omega_{e f f}=\frac{P_{e f f}}{\rho_{e f f}}$ satisfy the continuity equation in the same way as the general relativity

$$
\dot{\rho}_{e f f}^{(D E)}+3 H\left(1+\omega_{e f f}\right) \rho_{e f f}^{(D E)}=0 .
$$

Considering a power-law expansion on the brane, we set the scale factor to be $a(t)=a_{0} t^{h_{0}}$ with $h_{0}=1.2$. This choice is reliable from physical grounds since it is corresponding to an accelerated expansion on the brane. Figure 5 shows the behavior of the effective dark energy density versus the redshift for two signs of $\beta$. As this figure shows, although the effective energy density increases by decreasing $z$, its value remains positive only for negative values of $\beta$. This property is illustrated explicitly in figure $5(b)$. In this figure we have plotted $\rho_{\text {eff }}^{(D E)}$ versus $\beta$ for various values of the redshift. The acceptable range of $\beta$ can be deduced in a fascinating manner via imposing the null energy condition $\left(\rho_{\text {eff }}^{(D E)}+P_{\text {eff }}^{(D E)} \geq 0\right)$ for this DGP-inspired modified-GB scenario as we have shown in figure 6. The null energy condition is fulfilled for a narrow strip defined as $-0.25 \leq \beta \leq 0.05$. 

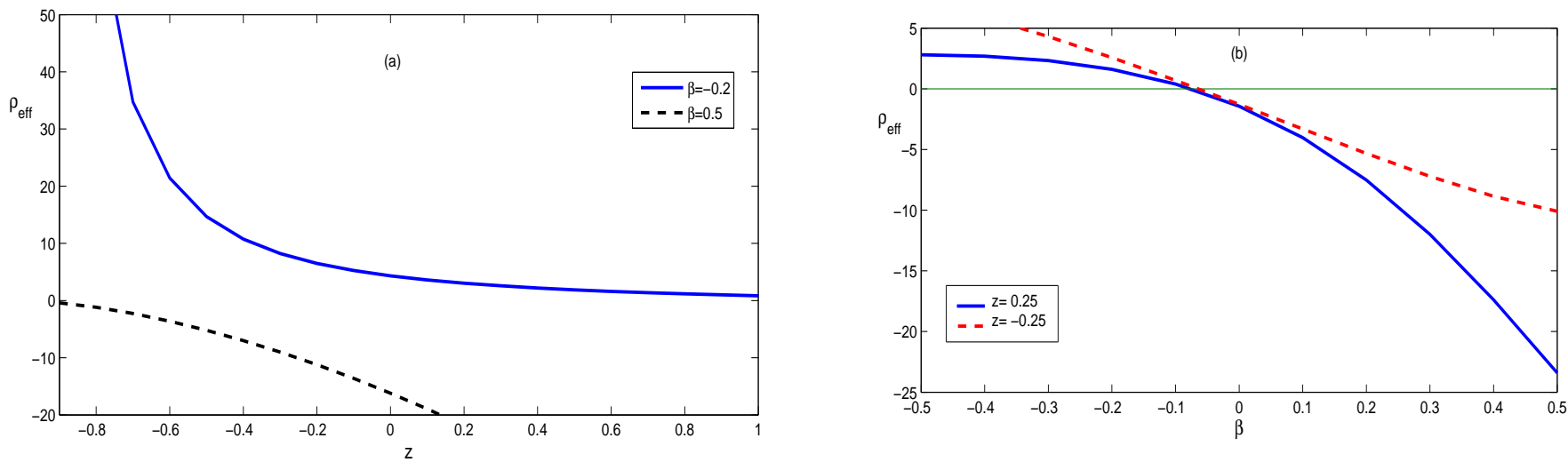

Figure 5: a) Variation of the effective energy density versus the redshift for $\beta=-0.2$ and $\beta=0.5$. In both cases $\rho_{\text {eff }}^{(D E)}$ increases by decreasing $z$, but its value always remains negative for positive values of $\beta$. b) Variation of $\rho_{\text {eff }}^{(D E)}$ versus $\beta$ for $z= \pm 0.25$. For positive values of $\beta$ the effective dark energy is always negative and the phantom-like prescription breaks down in this case.

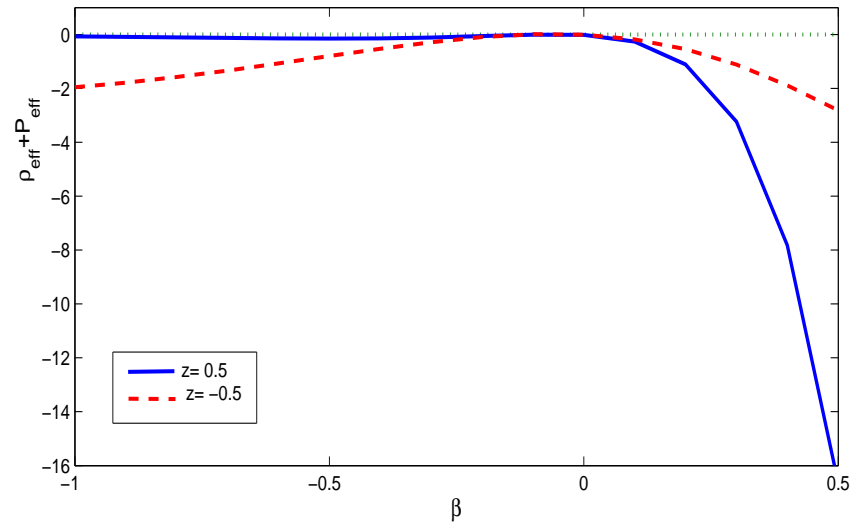

Figure 6: The null energy condition is fulfilled for a narrow strip $-0.25 \leq \beta \leq 0.05$. 
In figure $7(a)$, we have plotted the variation of the effective equation of state parameter versus the redshift for $\beta=-0.2$. Figure $7(b)$ shows that in the case of positive $\beta, \omega_{\text {eff }}$ is in the quintessence phase $\left(\omega_{\text {eff }}>-1\right)$. For negative values of $\beta$, the effective equation of state lies in the phantom phase with $\left(\omega_{\text {eff }}<-1\right)$. A unified treatment of the above results extracted from figures shows that an effective phantom-like behavior can be realized in this setup in the region $-0.25 \leq \beta \leq 0.05$. Therefore, a DGP-inspired modified Gauss-Bonnet gravity has the potential to realize a phantom-like behavior in appropriate subspaces of the model parameter space. It is important to note that with scale factor defined as $a=a_{0}\left(t_{s}-t\right)^{h_{0}}$, an effective phantom phase can be obtained also with negative $h_{0}$ ( see for instance [24] and references therein). However, in our setup an effective phantom phase is realized with a positive value of $h_{0}$ by virtue of the induced gravity effects.
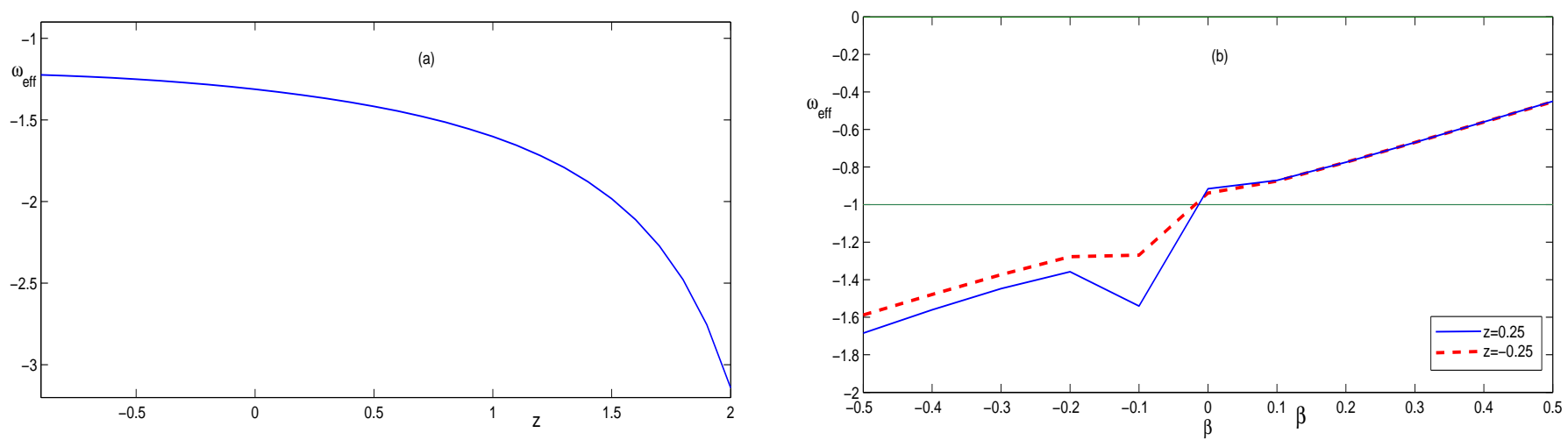

Figure 7: a) Variation of the effective equation of state parameter versus the redshift for $\beta=-0.2$ which remains in the phantom phase. b) Variation of the $\omega_{\text {eff }}$ versus $\beta$ for $z= \pm 0.25$. In the case of positive $\beta, \omega_{\text {eff }}$ is larger than -1 but for negative values of $\beta$ the effective equation of state lies in the phantom phase with $\omega_{e f f}<-1$.

\section{Summary and Conclusion}

In this paper we have studied possible realization of the phantom-like behavior in a DGPinspired scenario that curvature effects are taken into account by incorporation of the GaussBonnet term in the brane part of the action. Although the Gauss-Bonnet invariant essentially should be considered in the bulk action, we can introduce it on the brane by considering a coupling between this invariant and a scalar field on the brane. In this viewpoint, one can consider possible modification of the induced gravity on the brane by incorporation of higher order curvature terms such as the Gauss-Bonnet type terms. Then, we have investigated the 
phantom-like nature of this scenario with details. By the phantom-like behavior, we mean an effective energy density which is positive and grows with time and its equation of state parameter stays always less than -1 , that is, the effective equation of state parameter is in the phantom region of the parameter space $\left(\omega_{e f f}^{D E}<-1\right)$. In this paper, firstly we have considered the normal branch of the DGP-inspired Scalar-Gauss-Bonnet gravity ( which is ghost free) and we have explored the cosmological implications of the model. Especially, we have shown that this branch, which is not self-accelerating in a pure DGP setup, can account for a self-accelerating behavior at the late times due to the Gauss-Bonnet curvature effect. As another important outcome of this setup, we have shown that an effective phantom-like behavior can be realized without need to introduce phantom fields neither on the brane nor in the bulk. The effective equation of state parameter of the model has a smooth crossing of the cosmological constant line at the near past and in the same way as observations indicate. In the last stage, we have considered a DGP-inspired model with modified-GB term in the brane action. In this case, the brane part of the action is dynamically equivalent to the Scalar-Gauss-Bonnet gravity with vanishing kinetic-energy term. We have shown that this scenario can also realize a phantom-like behavior by adopting cosmologically viable ansatz. The phantom-like prescription is realized in this setup for appropriate subspaces of the model parameter space ( for instance with $-0.25 \leq \beta \leq 0.05$ ). It is important to note that in the absence of the induced gravity effect, an effective phantom phase has been obtained with negative powers of the scale factor, that is, a phantom ansatz with $h_{0}<0$ in $a=a_{0}\left(t_{s}-t\right)^{h_{0}}$. Here we have shown that the presence of an induced gravity term on the brane leads to an effective phantom-like behavior with a positive values of $h_{0}$ which we call it a quintessence ansatz. Finally, we have shown that the luminosity distance of the DGP-inspired scenario with curvature correction is closer to the $\Lambda$ CDM model than the $\Lambda$ DGP scenario. However, this result is dependent on the choice of the model parameter space. Since the $\Lambda$ CDM has very good agreement with observational data, our analysis shows that with a suitable choice of the parameter space of the model, the DGP-inspired $F(G, \phi)$ scenario has better agreement with recent observation than $\Lambda$ DGP. This result seems to be reasonable since the DGP-inspired $F(G, \phi)$ scenario has wider parameter space than the $\Lambda$ DGP model.

\section{References}

[1] A. G. Riess et al, Astrophys. J. 607 (2004) 665; W. M. Wood-Vasey et al, Astrophys. J. 666 (2007) 694-715 [arXiv:astro-ph/0701041]; D. N. Spergel et al, Astrophys. J. Suppl 170 (2007) 377; G. Hinshaw et al, Astrophys. J. Suppl., 288 (2007) 170; M. Colless et al, Mon. Not. R. Astron. Soc. 328 (2001) 1039; M. Tegmark et al, Phys. Rev. D 69 (2004) 103501; V. Springel, C. S. Frenk, and S. M. D. White, Nature (London) 440 (2006) 1137; S. P. Boughn and R. G. Crittenden, Mon. Not. R. Astron. Soc. 360 (2005) 1013; P. Fosalba and E. Gaztanaga, Mon. Not. R. Astron. Soc. 350 (2004) L37; J. D. McEwen et al, Mon. Not. R. Astron. Soc. 376 (2007) 1211; M. R. Nolta, Astrophys. 
J., 608 (2004) 10, P. Vielva et al, Mon. Not. R. Astron. Soc. 365 (2006) 891; C. R. Contaldi, H. Hoekstra and A. Lewis, Phys. Rev. Lett. 90 (2003) 221303.

[2] E. J. Copeland, M. Sami and Shinji Tsujikawa, Int. J. Mod. Phys. D 15 (2006) 1753, [ arXiv:hep-th/0603057]; N. Straumann, [arXiv:astro-ph/0009386]; Y. Fujii, Phys. Rev. D 62 (2000) 064004; L. P. Chimento, A. S. Jakubi and D. Pavon, [arXiv:astro-ph/0010079]; D. T. Valentini and L. Amendola Phys. Rev. D 65 (2002) 063508; N. Arkani-Hamed, P. Creminelli, S. Mukohyama and M. Zaldarriaga, JCAP 0404 (2004) 001; F. Piazza and S. Tsujikawa, JCAP 0407 (2004) 004; P. P. Avelino, Phys. Lett. B 611 (2005) 15; R. Curbelo T. Gonzalez and I. Quiros, Class. Quant. Grav. 23 (2006) 1585 [arXiv:astro-ph/0502141]; B. Hu and Y. Ling, Phys. Rev. D 73 (2006) 123510 [arXiv:hep-th/0601093]; P. B. Almeida and J. G. Pereira, Phys. Lett. B 636 (2006) 75; H. Wei and R. G. Cai, Phys. Rev. D 73 (2006) 083002; J. Kujat, R. J. Scherrer and A. A. Sen, Phys. Rev. D 74 (2006) 083501; P. P. Avelino, C. J. A. P. Martins, N. J. Nunes and K. A. Olive, Phys. Rev. D 74 (2006) 083508, [arXiv:astro-ph/0605690]; M. Kunz, L. Amendola and D. Sapone [arXiv:0806.1323]; J. B. Jimenez and A. L. Maroto, [arXiv:astro-ph/0812.1970]; X. M. Chen, Y. Gong, and E. N. Saridakis, [arXiv:grqc/0812.1117]; K. Nozari, M. R. Setare, T. Azizi and N. Behrouz, [arXiv:0810.1427]; M. Quartin, M. O. Calvao, S. E. Joras, R. R. R. Reis and I. Waga, JCAP 05(2008)007; P. Moyassari and M. R. Setare, Phys. Lett. B 674 (2009) 237, [ arXiv:0806.2418].

[3] P. J. E. Peebles and B. Ratra, Rev. Mod. Phys. 75 (2003) 559; V. Sahni, The Physics of the Early universe, edited by E. Papantonopoulos (Springer, New York 2005), P. 141 ; T. Padmanabhan, Proc. of the 29th Int. Cosmic Ray Conf. 10 (2005) 47.

[4] F. Perrotta, C. Baccigalupi and S. Matarrese, Phys. Rev. D. B61 (2000) 023507; B. Boisseau, G. Esposito-Farese, D. Polarski and A. A. Starobinsky, Phys. Rev. Lett. 85 (2000) 2236.

[5] S. Capozziello, V. F. Cardone, S. Carloni and A. Troisi, Int. J. Mod. Phys. D 12 (2003) 1969-1982, [arXiv:astro-ph/0307018]; S. Carroll et al. Phys. Rev. D 70 (2004) 043528; S. Nojiri and S. D. Odintsov, AIP Conf. Proc. 1115 (2009) 212-217, [arXiv:0810.1557]; T. P. Sotiriou and V. Faraoni, [ arXiv:/0805.1726]; A. A. Starobinsky, JETP. Lett. 86(2007) 157, [arXiv:/0706.2041]; F. S. N. Lobo, [arXiv:gr-qc/0807.1640]; T. Multamaki and I. Vilja, Phys. Rev. D73 (2006) 024018, [arXiv:astro-ph/0506692]; K. Nozari and M. Pourghassemi, JCAP 10 (2008) 044, [arXiv:0808.3701]; M. R. Setare, Int. J. Mod. Phys. D 17 (2008) 2219, [arXiv:0901.3252].

[6] G. Dvali, G. Gabadadze and M. Porrati, Phys. Lett. B 485 (2000) 208, [hep-th/0005016].

[7] D. J. Gross and J. H. Sloan, Nucl. Phys. B 291 (1987) 41; C. Charmousis and J. F. Dufaux, Class. Quant. Grav. 19 (2002) 4671, [arXiv:hepth/ 0202107]; S. C. Davis, Phys. Rev. D 67 (2003) 024030, [arXiv:hep-th/0208205]; P. Binetruy, C. Charmousis, S. C. 
Davis and J. F. Dufaux, Phys. Lett. B 544 (2002) 183, [arXiv:hep-th/0206089]; J. E. Lidsey and N. J. Nunes, Phys. Rev. D 67 (2003) 103510, [arXiv:astroph/ 0303168]; A. N. Aliev, H. Cebeci and T. Dereli, Class. Quant. Grav. 23 (2006) 591 [arXiv:hepth/0507121]; R. G. Cai, Y. G. Gong and B. Wang, JCAP 0603 (2006) 006, [arXiv:hepth/0511301]; R. G. Cai, H. Sh. Zang and A. Wang, Common. Theor. Phys 44 (2005) 948, [arXiv:hep-th/0505186].

[8] C. Deffayet, Phys. Lett. B 502 (2001) 199; C. Deffayet, G. Dvali and G. Gabadadze, Phys. Rev. D 65 (2002) 044023; C. Deffayet, S. J. Landau, J. Raux, M. Zaldarriaga and P. Astier, Phys. Rev. D 66 (2002) 024019; Kei-ichi Maeda, S. Mizuno and T. Torii, Phys. Rev. D 68 (2003)024033, [arXiv:gr-qc/0303039]; A. Lue and G. D. Starkman, Phys. Rev. D 70 (2004) 101501, [arXiv:astro-ph/0408246].

[9] K. Koyama, Class. Quantum Grav. 24 (2007) R231, [arXiv:0709.2399]; C. de Rham and A. J. Tolley, JCAP 0607 (2006) 004, [arXiv:hep-th/0605122].

[10] I. Antoniadis, J. Rizos and K. Tamvakis, Nucl. Phys. B 415 (1994) 497; S. Kawai, M. A. Sakagami and J. Soda, Phy. Lett. B 437 (1998) 284, [arXiv:gr-qc/9802033]; S. Kawai and J. Soda, Phy. Lett. B 460 (1999) 41, [arXiv:gr-qc/9903017];P. Kanti, J. Rizos and K. Tamvakis, Phys. Rev. D 59 (1999) 083512; N. E. Mavromatos and J. Rizos, Phys. Rev. D 62 (2000) 124004; T. Koivisto and D. F. Mota, Phys. Lett. B 644 ( 2007) 104, [astro-ph/0606078]; T. Koivisto and D. F. Mota, Phys. Rev. D 75 (2007) 023518, [hep-th/0609155]; M. Satoh, S.Kanno and J. Soda, Phys. Rev. D 77 (2008) 023526, [arXiv:0706.3585]; M. Satoh and J. Soda, JCAP 0809 (2008) 019, [arXiv:0806.4594].

[11] J. Callan et al, Nucl. Phys. B262 (1985) 593; D. J. Gross and J. H. Sloan, Nucl. Phys. B 291 (1987) 41; R. R. Metsaev and A. A. Tseytlin, Phys. Lett. B B191 (1987) 354; M. C. Bento and O. Bertolami, Phys. Lett. B B368 (1995) 198.

[12] S. Nojiri, S. D. Odintsov and M. Sasaki, Phys. Rev. D71 (2005) 123509.

[13] S. Nojiri, and S. D. Odintsov, Int. J. Geom. Meth. Mod. Phys. 4 (2007) 115; G. Cognola, E. Elizalde, S. Nojiri, S. D. Odintsov and S. Zerbini, Phys. Rev. D 73 (2006) 084007; S. Nojiri, and S. D. Odintsov, Phys. Lett. B 631 (2005) 1; S. Nojiri, S. D. Odintsov and M. Sami, Phys. Rev. D 74, 046004 (2006); S. Nojiri and S. D. Odintsov, J. Phys. Conf. Ser. 66, 012005 (2007); S. Capozziello, E. Elizalde, S. Nojiri and S. D. Odintsov, Phys. Lett. B 671, 193 (2009);C. G. Bohmer and F. N. Lobo, [arXiv:gr-qc/0902.2982v3].

[14] U. Seljak et al., 2005 Phys. Rev. D 71 [astro-ph/0407372] 103515.

[15] E. Komatsu et al. [WMAP Collaboration], Astrophys. J. Suppl. 180 (2009) 330, [arXiv:0803.0547].

[16] S. Weinberg, Rev. Mod. Phys. 61 (1989) 1; S. M. Carroll, Living Rev. Relativity 4 (2001) 1; R. Caldwell, R. Dave, P. Steinhardt, Phys. Rev. D 59 (1999) 123504. 
[17] R.R. Caldwell, Phys. Lett. B 545 (2002) 23 [astro-ph/9908168]; G. W. Gibbons, [arXiv:hep-th/0302199].

[18] R. R. Caldwell, M. Kamionkowski and N. N. Weinberg, Phys. Rev. Lett. 91 (2003) 071301, [arXiv:astro-ph/0302506]; F. Piazza and S. Tsujikawa, JCAP 0407 (2004) 004; S. M. Carroll, M. Hoffman and M. Trodden, Phys. Rev. D 68 (2003) 023509 [arXiv:astroph/0301273]; J. M. Cline, S. Jeon and G. D. Moore, Phys. Rev. D 70 (2004) 043543, [arXiv:hep-ph/0311312].

[19] L. P. Chimento, R. Lazkoz, R. Maartens and I. Quiros, JCAP 0609 (2006) 004, [arXiv:astro-ph/0605450]; R. Lazkoz, R. Maartens and E. Majerotto, Phys. Rev. D 74 (2006) 083510, [arXiv:astro-ph/0605701]; R. Maartens and E. Majerotto, Phys. Rev. D 74 (2006) 023004, [arXiv:astro-ph/0603353]. See also M. Bouhmadi-Lopez and P. Vargas Moniz, Phys. Rev. D 78 (2008) 084019, [arXiv:0804.4484]; M. Bouhmadi-Lopez, Nucl. Phys. B 797 (2008) 78-92, [arXiv:astro-ph/0512124]; M. Bouhmadi-Lopez and A. Ferrera, JCAP 0810 (2008) 011, [arXiv:0807.4678]; K. Nozari and F. Kiani, JCAP 07 (2009) 010, [ arXiv:0906.3806]; M. Bouhmadi-Lopez, [arXiv:0905.1962].

[20] S. Nojiri and S. D. Odintsov, Phys. Rev. D68 (2003) 123512, [arXiv:hep-th/0307288]; S. Nojiri and S. D. Odintsov, Class. Quantum Grav. 22 (2005) L35, [arxiv:hep-th/0409177]; S. Nojiri and S. D. Odintsov, Phys. Rev. D 74 (2006) 086009, [arxiv:hep-th/0611071]; K. Bamba, S. Nojiri and S. D. Odintsov, Phys. Rev. D. 79 (2009) 083014, [arXiv:0810.4296]; S. Nojiri and S. D. Odintsov, Phys. Rev. D. 78 (2008) 046006, [arXiv:0804.3519]; K. Bamba, S. Nojiri and S. D. Odintsov, JCAP 0810 (2008) 045, [arXiv:0807.2575]; K. Nozari and T. Azizi, [arXiv:0909.0351].

[21] J. W. York, Phys. Rev. Lett. B28 (1972) 1082; G. W. Gibbons and S. W. Hawking, Phys. Rev. D B15 (1977) 2752.

[22] V. Sahni and Y. Shtanov, JCAP 0311 (2003) 014, [arXiv:astro-ph/0202346]; V. Sahni, [arXiv:astro-ph/0502032].

[23] A. Lue and G. D. Starkman, Phys. Rev. D 70 (2004) 101501, [arXiv:astro-ph/0408246].

[24] G. Cognola, E. Elizalde, S. Nojiri, S. D. Odintsov and S. Zerbini, Phys. Rev. D 73 (2006) 084007, [arXiv:hep-th/0611198v3]. 